\title{
Simulation of Transient Mechanical Wave Propagation in Heterogeneous Soils
}

\author{
Arnaud Mesgouez, Gaëlle Lefeuve-Mesgouez, and André Chambarel \\ UMR A Climate, Soil and Environment, \\ Université d'Avignon et des Pays de Vaucluse, \\ INRA, Domaine St-Paul, F-84914 Avignon Cedex 9, France \\ arnaud.mesgouez@univ-avignon.fr
}

\begin{abstract}
A Finite Element Method using a $\mathrm{C}++$ code is developed to study the mechanical wave propagation in saturated porous soils. The modelization uses the complete Biot theory including the couplings between the solid and fluid phases. A matrix-free algorithm and a selection data technique are implemented in the code. Researches are focused on homogeneous and heterogeneous semi-infinite media in the case of transient regimes. The time domain results present the displacements over and within the half-space. In particular, we will see that the fluid wave front is strongly dependent on the proportion of heterogeneities in the ground.
\end{abstract}

\section{Introduction}

The study of the mechanical wave propagation in porous media is a subject of great interest in diverse scientific fields ranging from environmental engineering or vibration isolation to geomechanics. A saturated porous medium is a medium that presents on the microscopic spatial scale a solid deformable skeleton and a porous space filled with a viscous fluid. A macroscopic formulation can be deduced from the microscopic approach by homogenization: in this case, the medium is considered as a two-phase continuum. Biot's articles (see [1] and [2]) and Bourbié et al.'s review [3] are works of reference for the macroscopic mechanical wave propagation theory. In such a medium, three body waves exist: the $\mathrm{P} 1$ and $\mathrm{P} 2$ compressional waves and the $\mathrm{S}$ shear wave. The first compressional wave is a wave said to be quick whereas the second compressional wave is said to be slow and strongly attenuated. Moreover, for a semi-infinite medium, a surface wave also exists denoted as the Rayleigh $\mathrm{R}$ wave. Theoretical works are restricted to simple geometries. Consequently, they have to be completed by numerical approaches such as Finite Element or Boundary Element Methods, allowing the study of more complex problems to better modelize the ground. The difficult study of transient regimes has been treated numerically for specific cases by several authors: Zienkiewicz and Shiomi [4], Simon et al. [5] and Gajo et al. 6] for instance. In this paper, the authors propose a Finite Element Method of the whole Biot's equations in the case of homogeneous and heterogeneous soils. An efficient and accurate $\mathrm{C}++$ code is developed to deal with this problematic. 


\section{$2 \quad$ Mechanical Model}

\subsection{Governing Equations}

The theoretical approach is formulated using the Biot model: writing $u_{i}$ and $U_{i}$ respectively the solid and fluid displacement components, with usual notations, Biot's equations can be written as follows

$$
\begin{gathered}
\sigma_{i j, j}=(1-\phi) \rho_{s} \ddot{u}_{i}+\phi \rho_{f} \ddot{U}_{i} \\
p_{, i}=-\frac{\phi}{K}\left(\dot{U}_{i}-\dot{u}_{i}\right)+\rho_{f}(a-1) \ddot{u}_{i}-a \rho_{f} \ddot{U}_{i} \\
\sigma_{i j}=\lambda_{0 v} \varepsilon_{k k} \delta_{i j}+2 \mu_{v} \varepsilon_{i j}-\beta p \delta_{i j} \\
-\phi\left(U_{k, k}-u_{k, k}\right)=\beta u_{k, k}+\frac{1}{M} p
\end{gathered}
$$

The soil's characteristics are: $\lambda_{0 v}$ and $\mu_{v}$ (drained Lamé constants for the purely viscoelastic equivalent porous media), $\rho_{s}$ and $\rho_{f}$ (solid grains and fluid densities), $\phi$ (porosity), $K$ (hydraulic permeability), $a$ (tortuosity), $M$ and $\beta$ (Biot coefficients).

(11) and (2) are the motion equations, (3) and (41) are the constitutive relationships including a physical viscoelastic hysteretic Rayleigh damping of the soil.

\subsection{Finite Element Formulation and Numerical Resolution}

In order to obtain a first order time differential system, the solid and fluid particles' velocities are introduced: $v_{i}=\dot{u}_{i}$ and $V_{i}=\dot{U}_{i}$. Then, some algebraic manipulations are done to yield in the end a diagonal mass matrix. The weighted residual method with Galerkin ponderation gives integral forms (5) and (6) defined in the vector space $V(f)=[0, T] \times\left\{\frac{\partial f}{\partial t} \in L^{2}(\Omega), f \in H_{1}(\Omega)\right\}$ where $T$ and $(\Omega)$ are respectively the study time and the study space.

$$
\begin{aligned}
& \int_{\Omega} a \sigma_{i j, j} \delta v_{i} d \Omega+\int_{\Omega} \phi p_{, i} \delta v_{i} d \Omega=\int_{\Omega}\left[a(1-\phi) \rho_{s}+\phi(a-1) \rho_{f}\right] \dot{v}_{i} \delta v_{i} d \Omega \\
&+\int_{\Omega} \frac{-\phi^{2}}{K} V_{i} \delta v_{i} d \Omega+\int_{\Omega} \frac{\phi^{2}}{K} v_{i} \delta v_{i} d \Omega \\
& \int_{\Omega} \rho_{f}(a-1) \sigma_{i j, j} \delta V_{i} d \Omega-\int_{\Omega}(1-\phi) \rho_{s} p_{, i} \delta V_{i} d \Omega=\int_{\Omega} \frac{(1-\phi) \rho_{s} \phi}{K} V_{i} \delta V_{i} d \Omega \\
&+\int_{\Omega} \frac{-(1-\phi) \rho_{s} \phi}{K} v_{i} \delta V_{i} d \Omega+\int_{\Omega}\left[\rho_{f}^{2}(a-1) \phi+\rho_{s} \rho_{f} a(1-\phi)\right] \dot{V}_{i} \delta V_{i} d \Omega
\end{aligned}
$$

The weak formulation is then transformed using the Green theorem that introduces the boundary conditions. Then, for respectively two-dimensional and three-dimensional problems, triangular and tetrahedral linear isoparametric elements are used to mesh the $(\Omega)$ space. Afterwards, we can get an approximative solution of the second formulation with analytical discretization of the displacement and velocity components. 
For an elementary space (subscript ()$\left._{e}\right)$, the discrete system can be synthesized in the following form

$$
\begin{aligned}
& \sum_{e}\left\langle\delta W_{e}\right\rangle\left\{\left[\begin{array}{cccc}
{\left[m_{e}\right]} & 0 & 0 & 0 \\
0 & {\left[m_{e}\right]} & 0 & 0 \\
0 & 0 & {\left[m_{s e}\right]} & 0 \\
0 & 0 & 0 & {\left[m_{f e}\right]}
\end{array}\right]\left\{\dot{W}_{e}\right\}\right. \\
& \left.\left[\begin{array}{cccc}
0 & 0 & -\left[m_{e}\right] & 0 \\
0 & 0 & 0 & -\left[m_{e}\right] \\
{\left[k_{s e}\right]} & {\left[k_{s f e}\right]} & {\left[c_{s e}\right]} & -\left[c_{s e}\right] \\
{\left[k_{f s e}\right]} & {\left[k_{f e}\right]} & {\left[c_{f e}\right]} & -\left[c_{f e}\right]
\end{array}\right]\left\{W_{e}\right\}\right\}=\sum_{e}\left\langle\delta W_{e}\right\rangle\left\{F_{e}\right\}
\end{aligned}
$$

where $\left\{W_{e}\right\}=\left\langle\left\{u_{i}\right\}^{n}\left\{U_{i}\right\}^{n}\left\{v_{i}\right\}^{n}\left\{V_{i}\right\}^{n}\right\rangle^{t}$ is the elementary vector of the variables and $\left\{F_{e}\right\}$ the elementary vector of the load.

The assembling procedure leads to the following global differential system (superscript ()$\left.^{(G)}\right)$ with a diagonal global mass matrix

$$
[M] \frac{d}{d t}\left\{W^{(G)}\right\}+[K]\left\{W^{(G)}\right\}=\left\{F^{(G)}\right\}
$$

The time integration algorithm is a backward difference method modified with an upward time parameter $\alpha$, and is presented as follows for a $k$-order, $\Psi$ being the global residuum

$$
\begin{aligned}
& \text { While }\left(t_{n} \leq t_{\text {max }}\right) \\
& \left\{\begin{array}{c}
\left\{\Delta W_{n}^{i}\right\}=\Delta t_{n} \sum_{j=0}^{k-1} \lambda_{j}\left[M_{n-j}^{i}\right]^{-1} \times \\
\quad\left\{\Psi_{n-j}\left(\left\{W_{n-j}\right\}+\alpha_{j}\left\{\Delta W_{n-j}^{i-1}\right\}, t_{n}+\alpha_{j} \Delta t_{n}\right)\right\} \\
i=1,2 \ldots \text { until }\left\|\left\{\Delta W_{n}^{i}\right\}-\left\{\Delta W_{n}^{i-1}\right\}\right\| \leq \text { tolerance }
\end{array}\right\} \\
& \left\{W_{n+1}\right\}=\left\{W_{n}\right\}+\left\{\Delta W_{n}\right\} \\
& t_{n+1}=t_{n}+\Delta t_{n} \\
& \text { end while }
\end{aligned}
$$

It requires inner iterations for each time step until the tolerance criterium is reached.

\subsection{Structure of the Code}

The Finite Element $\mathrm{C}++$ code is organized in three classes: element, elementary matrices and building-resolution classes. Thus, three objects connected by a single heritage are constructed and they form a solver. In practise, we obtain very low sized solvers (less than $1000 \mathrm{C}++$ lines).

In this code, the mass and stiffness matrices are never built because a matrixfree technique is used. As the global mass matrix is diagonal, its inversion is an easy process. The size of the global vector of unknowns is optimized by the use of an expert multigrid system called AMS (see 7] and [8]). On the whole, a high performance level is obtained both in terms of CPU and storage costs. 


\section{$3 \quad$ Results}

\subsection{Half-Space Model}

A vertical impulse ( $y$ axis) load acts over the free surface ( $x$ axis) of a twodimensional half-space. The soil characteristics are relative to a porous stiff ground: the dimensionless values are given in Table 11, In all the following section, the numerical values are dimensionless, using three independent mechanical quantities. Dirichlet's conditions corresponding with the zero displacements for both phases are imposed at depth at the border of the medium. The $(\Omega)$ space is meshed with 50626 triangular elements and 25617 eight-degrees-of-freedom nodes.

Table 1. Dimensionless soil parameters and associated celerity values

\begin{tabular}{cccccccccccccccc}
\hline$\lambda_{0}$ & $\mu$ & $K_{s}$ & $K_{f}$ & $M$ & $\beta$ & $\rho_{s}$ & $\rho_{f}$ & $a$ & $K$ & $\phi$ & $\eta$ & $c_{P 1}$ & $c_{P 2}$ & $c_{S}$ & $c_{R}$ \\
0.56 & 0.83 & 4 & 0.22 & 0.53 & 0.72 & 1 & 0.39 & 1.5 & 0.26 & 0.4 & 0.01 & 1.9 & 0.65 & 1.1 & 1.05 \\
\hline
\end{tabular}

\section{$3.2 \quad$ Homogeneous Case}

Figure 1(a) presents the horizontal solid displacements on the surface of the half-space $(-3<x<3, y=0)$ versus time variable $(0<t<2)$. The $\mathrm{P} 1$ (respectively $\mathrm{R}$ ) contribution is focused on a line the slope of which corresponds to the P1 (resp. R) celerity. The $\mathrm{S}$ wave is lost to the eye due to the dominating Rayleigh wave: its contribution could be seen when in vertical displacements. The P2 wave carries very little energy and is mixed with the whole surface displacement which here prevents us from seeing it. The P1 and R waves give opposite contributions with a preponderant part to Rayleigh's. Moreover, in the case of a vertical sollicitation as studied here, the horizontal displacement is an odd function of $\mathrm{x}$ variable, whereas the vertical displacement is even. Also note, from the same figure, that the displacement decreases geometrically in addition to the physical damping. In Figure 1(b), which shows a section of Figure $1(\mathrm{a})$ for $x=1.5$, a visualization of the vertical displacement is added. The theoretical arrival times are underlined and the contribution of the $\mathrm{S}$ wave is clearly perceptible on the vertical displacement.

Figure 2(a) shows the variation of the fluid vertical displacements in depth under the load $(x=0)$ versus time variable. The Rayleigh wave decreases exponentially with depth. Consequently, the displacements underline the two compressional waves P1 and P2. On Figure 2(b) the two theoretical lines relative to each wave speed are plotted: a good agreement is obtained with the contour levels.

Figures 3 and 4 are plotted for $t=1.5$. The P1 wave front corresponds to a half-circle the radius of which equals 2.8: this is clearly perceptible on Figure 3. From the same figure, note that the Rayleigh wave gives a predominant contribution on the surface: this corresponds to the red areas beginning at $x=$ 1.6. Moreover, with the chosen parameters, the fluid and solid phases are strongly 


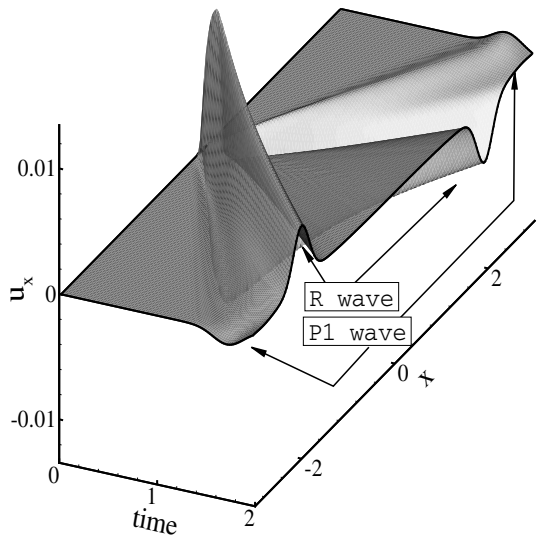

(a) Surface horizontal displacements

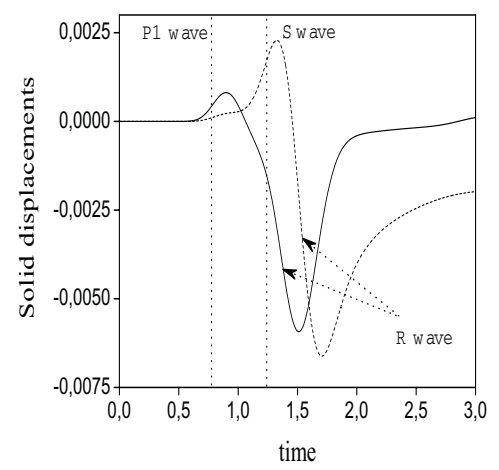

(b) Observational point $x=1.5$, horizontal (solid line) and vertical (dotted line) displacements

Fig. 1. Surface solid displacements versus time

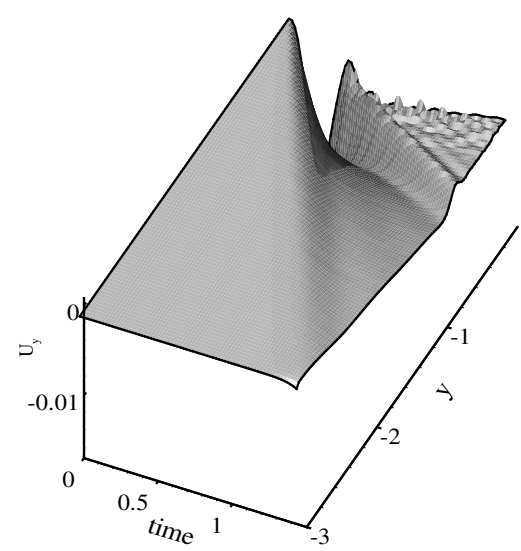

(a) In-depth visualization

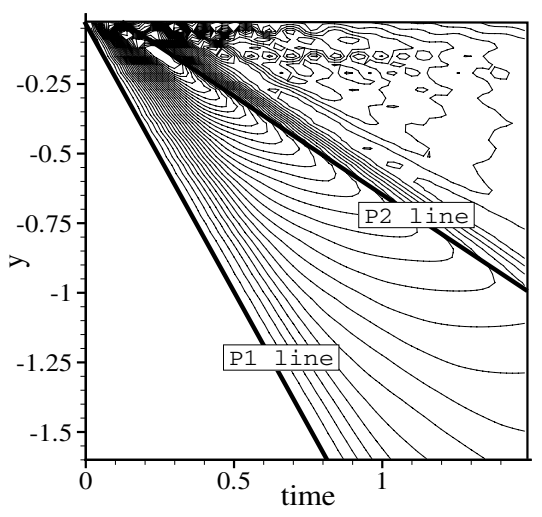

(b) Contour levels

Fig. 2. In-depth vertical fluid displacements versus time

uncoupled, thus the $\mathrm{S}$ wave exists only in the solid phase $(x=1.7)$. Due to the competition between the $\mathrm{R}$ and $\mathrm{S}$ waves which have neighboring speeds, the $\mathrm{S}$ wave front is more perceptible in depth.

For the fluid phase, Figure 4, red zones are caused by the P1 wave which presents a more widely spread time wave front. This analysis has been confirmed by a study of the displacements of time-related individual points positioned under the load. 


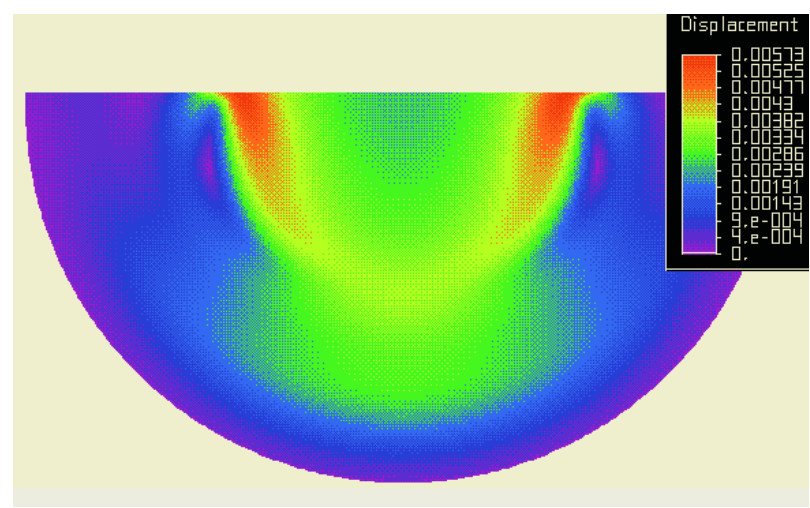

Fig. 3. Solid displacement field for a homogeneous medium at $t=1.5$

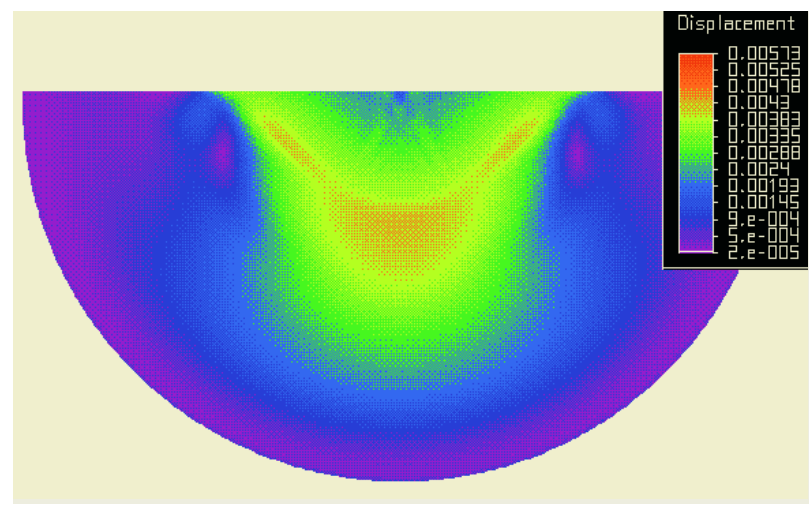

Fig. 4. Fluid displacement field for a homogeneous medium at $t=1.5$

\subsection{Heterogeneous Case}

The ground is constituted by a mixture of two kinds of media which are distributed randomly: the main part of this heterogeneous soil has the characteristics defined in Table 1. The second one represents softer inclusions $\left(\lambda_{0}=0.011\right.$, $\mu=0.011, M=0.271, \beta=0.995, \phi=0.6, \eta=1)$ and its proportion ranges from 1 percent to 40 percent.

In these cases, the solid wave fronts are on the whole less modified than the fluid ones, (see Figures 5 and [6). They remain well-ordered up to at least a 20 percent distribution. For a higher proportion, the solid wave fronts are dismembered and the contour levels are not concentric any more. Moreover, the inclusions focus the mechanical deformations over a smaller area in which the displacement values are higher. The presence of soft elements slows down the progression of the solid and fluid deformations in comparison with the purely homogeneous case.

As for the fluid phase, even for a low proportion of inclusions ( 1 or 5 percent), the wave fronts are disordered. Some areas present higher deformations due to 


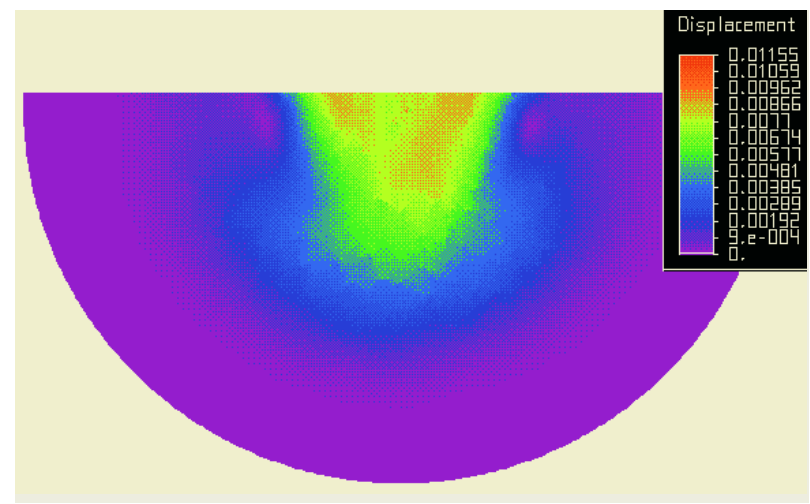

Fig. 5. Solid displacement field for a heterogeneous medium with $40 \%$ of inclusions at $t=1.5$

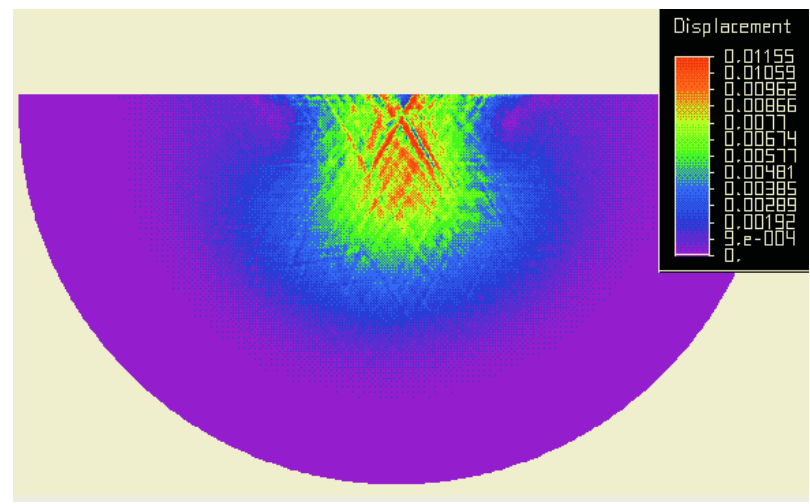

Fig. 6. Fluid displacement field for a heterogeneous medium with $40 \%$ of inclusions at $t=1.5$

the higher proportion of fluid in the softer inclusions. Moreover, fluid amplitudes, which are lower than solid ones in the homogeneous case, become equal if not higher with the inclusions. In conclusion, a deeper change occurs for the fluid phase.

\section{Conclusion and Further Works}

The study of the mechanical wave propagation has been carried out using a finite element method and a modified backward difference method for the time integration algorithm. A matrix-free algorithm and a selection data technique are implemented in the $\mathrm{C}++$ objet-oriented program. Different examples of homogeneous and heterogeneous semi-infinite media have been presented. Further works will include the parallelisation of the code and the study of three-dimensionnal geometries. 


\section{References}

1. Biot, M.A.: Generalized theory of acoustic propagation in porous dissipative media. J. Acoust. Soc. Am. 34(9) (1962) 1254-1264

2. Biot, M.A.: Mechanics of deformation and acoustic propagation in porous media. J. Appl. Phys. 33(4) (1962) 1482-1498

3. Bourbié, T., Coussy, O., Zinszner, R.: Acoustics of Porous Media. (1987) Paris: Editions Technip

4. Zienkiewicz, O.C., Shiomi, T.: Dynamic behaviour of saturated porous media: the generalized Biot formulation and its numerical solution. Int. J. Numer. Anal. Methods Geomech. 8 (1984) 71-96

5. Simon, B.R., Wu, J.S.S., Zienkiewicz, O.C., Paul, D.K.: Evaluation of $\mathrm{u}-\mathrm{w}$ and $\mathrm{u}-\pi$ finite element methods for the dynamic response of saturated porous media using one-dimensional models. Int. J. Numer. Anal. Methods Geomech. 10 (1986) 461-482

6. Gajo, A., Saetta, A., Vitaliani, R.: Evaluation of three and two field finite element methods for the dynamic response of saturated soil. Int. J. Numer. Anal. Methods Geomech. 37 (1994) 1231-1247

7. Chambarel, A., Ferry, E.: Finite Element formulation for Maxwell's equations with space dependent electric properties. Rev. Eur. Elém. Finis 9(8) (2000) 941-967

8. Chambarel, A., Bolvin, H.: Simulation of a compressible flow by the finite element method using a general parallel computing approach. Lect. Notes Comput. Sc., Springer-Verlag, Vol. 2329 (2002) 920-929 